\title{
Erratum to: ${ }^{18}$ F-Fluorocholine PET/CT for early response assessment in patients with metastatic castration-resistant prostate cancer treated with enzalutamide
}

\author{
Ugo De Giorgi ${ }^{1}$ - Paola Caroli ${ }^{2}$ Emanuela Scarpi ${ }^{3}$ - Vincenza Conteduca ${ }^{1}$. \\ Salvatore Luca Burgio ${ }^{1}$. Cecilia Menna ${ }^{1}$ - Andrea Moretti ${ }^{4} \cdot$ Riccardo Galassi $^{4}$. \\ Lorena Rossi $^{1} \cdot$ Dino Amadori $^{1} \cdot$ Giovanni Paganelli $^{2} \cdot$ Federica Matteucci $^{2}$
}

Published online: 22 April 2015

(C) Springer-Verlag Berlin Heidelberg 2015

\section{Erratum to: Eur J Nucl Med Mol Imaging DOI 10.1007/s00259-015-3042-5}

Owing to an error in typesetting, a table that should have been presented as part of Table 2 was separated from Table 2 and placed on an earlier page. The correct and complete Table 2 is reproduced here.

The online version of the original article can be found at http://dx.doi.org/ $10.1007 / \mathrm{s} 00259-015-3042-5$.

Ugo De Giorgi

ugo.degiorgi@irst.emr.it

1 Department of Medical Oncology, Istituto Scientifico Romagnolo per lo Studio e la Cura dei Tumori (IRST) IRCCS, via Maroncelli 40, 47014 Meldola, Italy

2 Diagnostic Nuclear Medicine Unit, Istituto Scientifico Romagnolo per lo Studio e la Cura dei Tumori (IRST) IRCCS, Meldola, Italy

3 Unit of Biostatistics and Clinical Trials, Istituto Scientifico Romagnolo per lo Studio e la Cura dei Tumori (IRST) IRCCS, Meldola, Italy

4 Nuclear Medicine Unit, Morgagni-Pierantoni Hospital, Forlì, Italy 
Table 2 Comparison of PSA decline and FCH PET/CT in nonprogressing vs progressing patients

\begin{tabular}{|c|c|c|c|}
\hline $\begin{array}{l}\text { Response at } \\
\text { Follow-up } \\
\text { FCH PET/CT Imaging } \\
\text { Study }\end{array}$ & $\begin{array}{l}\text { PSA Response } \\
\text { PSA } \\
\text { decline } \geq 50 \%\end{array}$ & $\begin{array}{l}\text { No PSA } \\
\quad \text { decline } \geq 50 \%\end{array}$ & Total \\
\hline NonProgression $^{\mathrm{a}}$ & $10(59 \%)$ & $7(41 \%)$ & $17(50 \%)$ \\
\hline Progression & $3(18 \%)$ & $14(82 \%)$ & $17(50 \%)$ \\
\hline Total & $13(38 \%)$ & $\begin{array}{l}21(62 \%) \\
\text { Lower }\end{array}$ & $\begin{array}{l}34(100 \%) \\
\text { Upper }\end{array}$ \\
\hline Measurement & Estimate & $95 \% \mathrm{CI}$ & $95 \% \mathrm{CI}$ \\
\hline Positive $\%$ Agreement & $67 \%$ & $48 \%$ & $81 \%$ \\
\hline Negative \% Agreement & $77 \%$ & $59 \%$ & $89 \%$ \\
\hline Positive Predictive Value & $82 \%$ & $65 \%$ & $93 \%$ \\
\hline Negative Predictive Value & $59 \%$ & $41 \%$ & $75 \%$ \\
\hline Overall Agreement & $71 \%$ & $55 \%$ & $86 \%$ \\
\hline Odds Ratio & 6.7 & 1.38 & 32.3 \\
\hline
\end{tabular}

${ }^{\text {a }}$ Including 7 pts with partial response (PR) and 10 with stable disease (SD) at FCH PET/CT, achieving a PSA response in 4/7 (57\%) PR and 6/10 (60 \%) SD cases, respectively

\begin{tabular}{lll}
\hline $\begin{array}{l}\text { Measurement } \\
\text { Agreement (\%) }\end{array}$ & Estimate & $95 \%$ CI \\
Positive & & \\
Negative & $67 \%$ & $48-81 \%$ \\
Predictive value & $77 \%$ & $59-89 \%$ \\
Positive & & \\
Negative & $82 \%$ & $65-93 \%$ \\
Overall agreement & $59 \%$ & $41-75 \%$ \\
Odds ratio & $71 \%$ & $55-86 \%$ \\
\end{tabular}

